\title{
High sensitivity 8-color flow cytometry assay for paroxysmal nocturnal hemoglobinuria granulocyte and monocyte detections
}

\author{
RAY CHUN-FAI CHAN, RICHARD H. LEUNG, ALBERT POSADAS, THOMAS S. LOREY and ALLISON J. SHAW
}

\author{
Department of Flow Cytometry, Kaiser Permanente Regional Laboratory Northern California, \\ The Permanente Medical Group, Inc., Berkeley, CA 94710, USA
}

Received October 24, 2017; Accepted December 12, 2017

DOI: $10.3892 /$ br.2018.1047

\begin{abstract}
Flow cytometry is the gold standard in diagnosis of paroxysmal nocturnal hemoglobinuria (PNH) by detecting the absence of glycol-phosphatidyl inositol (GPI)-linked protein expression on granulocyte and monocyte surfaces. However, the current assays are not optimized and require improvement, particularly in reducing background fluorescence and optimizing sensitivity and specificity. With more fluorochromes available and with advances in instrument engineering, rare populations may be identified with high sensitivity. The present study assessed an 8-color combination of comprehensive GPI-linked markers, namely fluorescein-labeled proaerolysin (FLAER), cluster of differentiation 157 (CD157), CD24 and CD14, and the lineage markers for granulocyte (CD15) and monocyte (CD64) cells to detect PNH clones. Additionally, to optimize the PNH flow assay, a 'dump' channel was used, comprised of CD5 and CD19, to exclude non-specific binding in order to reduce background. This method aimed to improve sensitivity and reduce the background to create an optimized PNH flow cocktail. The results demonstrated that the current 4-color PNH combination identifies a CD55 ${ }^{-}$and $\mathrm{FLAER}^{+}$population that is not PNH clones. By contrast, the 8-color panel delineated $\mathrm{PNH}$ clones from both monocyte and granulocytes by using granulocyte antigen (CD15) and monocyte antigen (CD64) as a gating strategy. The sensitivity was $0.01 \%$ for granulocytes and $0.05 \%$ for monocytes with an acquisition of 100,000 monocyte and granulocyte events. The background on a normal whole blood sample was $0.00076 \%$ on monocytes and $0.00277 \%$ on granulocytes. Thus, overall, the 8-color PNH assay exhibited high levels of specificity and sensitivity.
\end{abstract}

Correspondence to: Dr Ray Chun-Fai Chan, Department of Flow Cytometry, Kaiser Permanente Regional Laboratory Northern California, The Permanente Medical Group, Inc., 1725 Eastshore Highway, Berkeley, CA 94710, USA

E-mail: chun-fai.chan@kp.org

Key words: multicolor antibody cocktail, paroxysmal nocturnal hemoglobinuria, high sensitivity 8-color flow, fluorescein-labeled proaerolysin
The 8-color combination facilitated the improvement and enhancement of sensitivity in PNH clone identification, and may provide a useful tool for pathologists in $\mathrm{PNH}$ diagnosis and for monitoring patients at risk of developing classical/hemolytic PNH, to enable treatment to be delivered promptly.

\section{Introduction}

Multicolor flow cytometry provides detailed phenotypic information on the cellular composition of a sample. It allows for the identification of cellular subsets based on specific markers both on the surface of and inside cells (1). With the development of novel classes of fluorochromes and advancements in cytometer design, the use of more than 8 colors for flow cytometry should become common practice in clinical labs and increase the sensitivity of current assays (2).

Paroxysmal nocturnal hemoglobinuria $(\mathrm{PNH})$ is a rare stem cell disorder caused by acquired mutation in the X-linked phosphatidylinositol glycan complementation class A gene, with an annual incidence rate of 1.3 new cases per million of the population per year (3-8). The acquired mutation generates a defect in the expression of glycol-phosphatidyl inositol (GPI)-linked structures such as the complement regulatory proteins, CD55 and CD59 (9-12). A lack of these GPI-linked complement regulatory proteins on red blood cells (RBCs) may cause intravascular complement-mediated hemolysis, resulting in the release of free hemoglobin and leading to the symptoms of PNH, which may include the development of anemia, hemoglobinuria, thrombosis, dysphagia, abdominal pain, pulmonary hypertension, renal impairment and erectile dysfunction (4,13-16). Previous PNH assays have focused on loss of CD55 and CD59 on CD235a red cells or granulocytes $(17,18)$. However, pathological hemolysis of PNH red cells may generate inaccurate results, as many patients who are screened for PNH have received erythrocyte transfusions, which may contribute to underestimating the size of the $\mathrm{PNH}$ clone population. As a result, $\mathrm{PNH}$ erythrocytes may be undetectable even when a substantial PNH population is present (19). Furthermore, CD55 expression cannot distinguish intermediate complement sensitivity (type II) and more abnormal PNH-type III RBC clones (20), and the sensitivity of CD55 alone is not high enough to detect PNH population sizes of less than $1 \%$ (21). Despite there being therapeutic support 
Table I. Configuration of 8-color flow assay cocktail for paroxysmal nocturnal hemoglobinuria detection.

\begin{tabular}{lcccccccc}
\hline Fluorochrome & A488 & PE & PerCP & PE-Cy7 (dump) & APC & APC-A750 & V450 & V500-C \\
\hline Marker & FLAER & CD157 & CD14 & CD5+CD19 & CD24 & CD64 & CD15 & CD45 \\
Clone & FLAER & SY/11B5 & MfP9 & L17F12+SJ25C1 & ALB9 & 22 & MMA & 2D1 \\
Volume/test $(\mu 1)$ & 10 & 10 & 10 & $2.5+2.5$ & 5 & 5 & 2.5 & 5 \\
\hline
\end{tabular}

$\mathrm{CD}$, cluster of differentiation; FLAER, fluorescein-labeled proaerolysin; PE, phycoerythrin; APC, allophycocyanin; PerCP, peridinin chlorophyll protein complex; Cy-, cyanine; A-, Alexa Fluor.

for patients with PNH, including transfusions and anticoagulation management (22), the survival rate remains low, with $35 \%$ of cases being fatal within 5 years of diagnosis (23-25). Small PNH clones may grow over time and benefit from eculizumab treatment (25). Thus, high-sensitivity PNH assays are required to detect PNH populations of less than $1 \%$ in order to follow up closely and to provide early treatments for patients.

To improve the resolution of $\mathrm{PNH}$ detection, the present study formulated an 8-color combination for the detection of PNH white blood cells (WBCs), which included lineage-gating antibodies (CD64 for monocytes and CD15 for granulocytes) together with fluorescein-labeled proaerolysin (FLAER) and three GPI-linked markers, namely CD157 [both granulocyte and monocyte-specific (26)], CD24 [granulocyte-specific (27)] and CD14 [monocyte-specific (28)]. This combination matches the International Clinical Cytometry Society (ICCS) guidelines for the diagnosis and monitoring of $\mathrm{PNH}$ and related disorders by flow cytometry (20).

To the best of our knowledge, this present report is the first to identify a high-sensitivity 8-color antibody cocktail for PNH flow detection with the use of a dump channel. It may be a useful tool for PNH detection and has the potential to improve the accuracy, work flow, cost and diagnostic process while saving the labor and time of technicians and clinical pathologists.

\section{Materials and methods}

Blood samples and antibodies. Whole blood (WB) samples (100 $\mu \mathrm{l})$ from $\mathrm{PNH}$ patients were provided by the College of American Pathologists (CAP; Northfield, IL, USA) and UK NEQAS (London, UK) between May 2017 and August 2017. WB samples $(100 \mu \mathrm{l})$ from 10 healthy donors (aged 58.4 \pm 11.1 years) were also collected. The samples were stored in BD Vacutainer ${ }^{\mathrm{TM}}$ blood collection tubes containing EDTA as an anti-coagulant (BD Biosciences, Franklin Lakes, NJ, USA) at $4^{\circ} \mathrm{C}$ and tested within a month. For certain experiments, $\mathrm{PNH}$ proficiency test samples were obtained from CAP and UK NEQAS. Alexa Fluor (A)488-conjugated FLAER was purchased from Cedarlane (Burlington, ON, Canada). Phycoerythrin (PE)-conjugated anti-CD157 (SY/11B5), Allophycocyanin (APC)-conjugated anti-CD24 (ALB9) and APC-A750-conjugated anti-CD64 (clone 22) were purchased from Beckman Coulter, Inc. (Brea, CA, USA). Peridinin chlorophyll protein complex (PerCP)-conjugated anti-CD14 (MfP9), PE-cyanine (Cy)7-conjugated anti-CD5 (L17F12), PE-Cy7-conjugated anti-CD19 (SJ25C1), V450-conjugated
Table II. Target number of events for paroxysmal nocturnal hemoglobinuria populations with high sensitivity.

\begin{tabular}{lcc}
$\begin{array}{l}\text { No. of events (FLAER }{ }^{-} \text {- } \\
\text { CD157 } \text { on Grans) }\end{array}$ & Total events & Sensitivity (\%) \\
\hline 5 & 100,000 & 0.005 \\
10 & 100,000 & 0.010 \\
20 & 100,000 & 0.050 \\
\hline
\end{tabular}

anti-CD15 (MMA), V500-C-conjugated anti-CD45 (2D1), PerCP-Cy5.5-conjugated anti-CD3 (SK7), APC-conjugated anti-CD4 (SK3), APC-H7-conjugated anti-CD8 (SK1), V450-conjugated anti-CD7 (M-T701), PE-conjugated anti-CD55 (IA10) and APC-conjugated anti-CD14 (MfP9) were purchased from BD Biosciences. All the antibodies were titrated to optimize the cocktail performance. The present study was reviewed by the Research Determination Office of Kaiser Permanente (Oakland, CA, USA) and determined not to be human research due to the use of secondary de-identified samples; therefore, the requirements of informed patient consent and ethical approval were waived.

Cell staining with 8-color antibody cocktail and acquisition. The 8-color antibody cocktail for PNH detection was prepared according to the configuration listed in Table I. A total of $1 \times 10^{6}$ cells in the EDTA-whole blood samples were transferred to $12 \times 75 \mathrm{~mm}$ Falcon tubes and stained with the 8 -color cocktail antibody cocktail for $15 \mathrm{~min}$ at room temperature in dark. The whole blood was then lysed with $2 \mathrm{ml}$ BD FACS ${ }^{\mathrm{TM}}$ Lysing Solution (BD Biosciences) for $10 \mathrm{~min}$ at room temperature. After washing the cells twice with phosphate-buffered saline (PBS; Beckman Coulter, Inc.) and resuspending in $0.5 \mathrm{ml} 0.5 \%$ paraformaldehyde as fixation buffer (Polysciences, Inc., Warrington, PA, USA), data were acquired on a BD FACSCanto ${ }^{\mathrm{TM}}$ II flow cytometer with standard configuration (BD Biosciences). A total of 100,000 events in the monocyte and granulocyte cell gates were acquired. BD FACSDiva ${ }^{\mathrm{TM}}$ v.6.1.3 software under standard 8-color configuration (BD Biosciences) was used for acquisition and analysis. For compensation, a BD ${ }^{\mathrm{TM}}$ Comp Beads kit was used to create cocktail-specific compensation matrices on whole blood from the healthy donors utilizing batch-matched single color reagents following the manufacturer's instructions. For a 4-color control experiment, PE-CD55 (10 $\mu \mathrm{l})$, APC-CD14 $(5 \mu \mathrm{l})$, FLAER-A488 $(10 \mu \mathrm{l})$ 


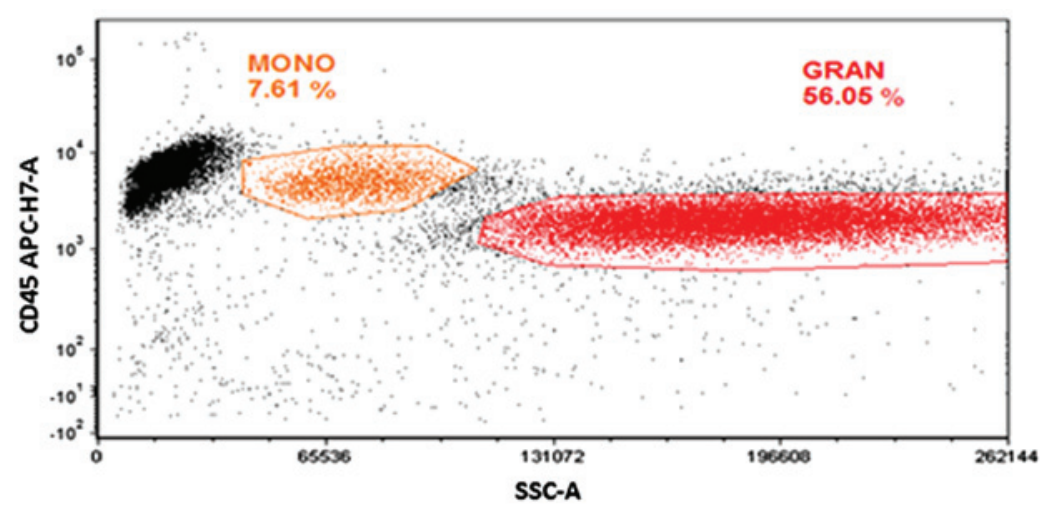

GRAII

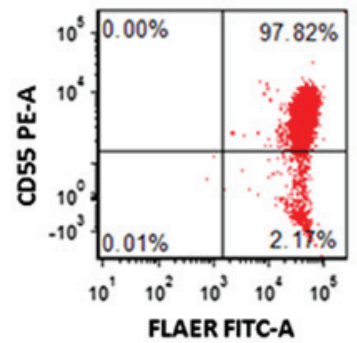

Mol10

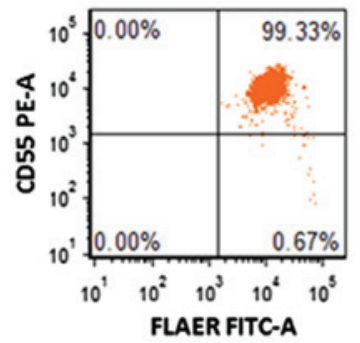

GRAII

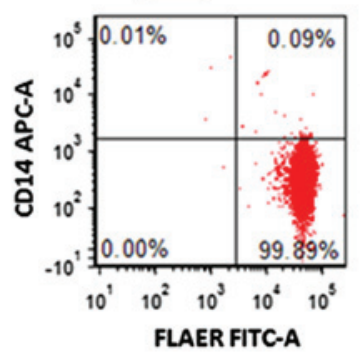

Mol10

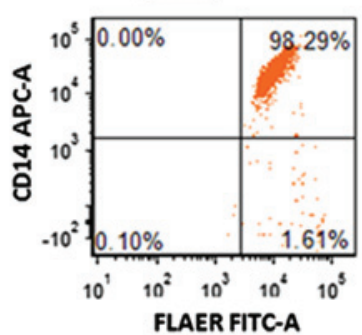

GRAII

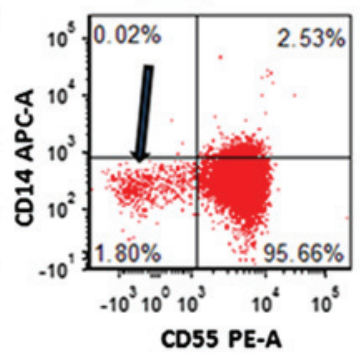

Mol10

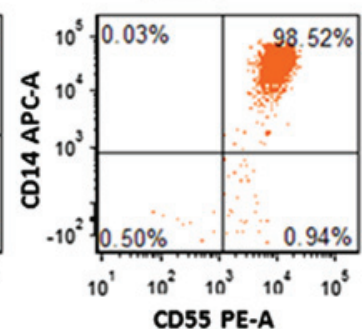

Figure 1. Paroxysmal nocturnal hemoglobinuria flow assay with a 4-color cocktail. Whole blood was stained with a 4-color cocktail (FLAERA488/CD55-PE/CD14-APC/CD45-V500-C) and lysed with FACSLyse. A total of 50,000 events were collected. Monocytes and granulocytes were gated on a CD45/SSC plot. The top panel exhibits the granulocytes on CD55/FLAER, CD14/FLAER and CD14/CD55 plots. The bottom panel exhibits the same plots with monocyte gating. The arrow indicates the CD55 population with FLAER ${ }^{+}$expression. Representative dot plots are shown $(n=3)$. MONO, monocyte; GRAN, granulocyte; CD, cluster of differentiation; FLAER, fluorescein-labeled proaerolysin; A488, Alexa Fluor 488; PE, phycoerythrin; APC, allophycocyanin; FITC, fluorescein isothiocyanate; SSC, side scatter; -A, area.

and V500-C-CD45 (5 $\mu \mathrm{l})$ were used to stain four normal whole blood samples (100 $\mu 1$ each). For certain experiments, whole blood was stained with PerCP-Cy5.5-CD3, APC-CD4, V450-CD7 and APC-H7-CD8.

Sensitivity test. The percentage of PNH clones [FLAER $/$ CD157- on $\mathrm{CD}^{-} 4^{+}$granulocytes (Grans)] was determined using the 8-color cocktail listed in Table I. Three PNH samples were spiked in $100 \mu \mathrm{l}$ normal whole blood to obtain a final concentration of PNH clones of $0.5-2 \%$. They were then serial diluted at 1:1 (v:v) using normal whole blood as diluent up to 0.005-0.01\% expected PNH percentage (Table II). Unspiked normal whole blood was used as a negative control. Following preparation of the serial dilutions, the samples were stained with the 8-color cocktail following the staining procedure described above. A total of 100,000 events in the monocyte and granulocyte gates were acquired for each sample. $\mathrm{CD}^{+} 5^{+}$Grans FLAER-/CD157- $\mathrm{PNH}$ clones were identified on CD157/FLAER dot plots.
Data acquisition. Data acquisition was performed with FCS Express 5 (De Novo Software, Glendale, CA, USA) and BD FACSDiva $^{\mathrm{TM}}$ v.6.1.3 using gating strategy on dot plots, after which appropriate subsets were identified.

Statistical analysis. To assess the correlation of $\mathrm{CD}^{+} 5^{+} \mathrm{Grans}$ FLAER-/CD157- ${ }^{-}$PH clones between expected and actual percentages, a linear regression analysis was performed. All statistical calculations were performed with Excel 2016 (Microsoft Corporation, Redmond, WA, USA) and Prism 7 (GraphPad Software, Inc., La Jolla, CA, USA). $\mathrm{R}^{2}>0.95$ and $\mathrm{P}$-value $<0.0001$ were considered to indicate significant correlation.

\section{Results}

Normal blood exhibits positive staining for CD55 using the 4-color PNH panel. In the present study, a whole blood sample from a healthy donor was stained with a 4-color 

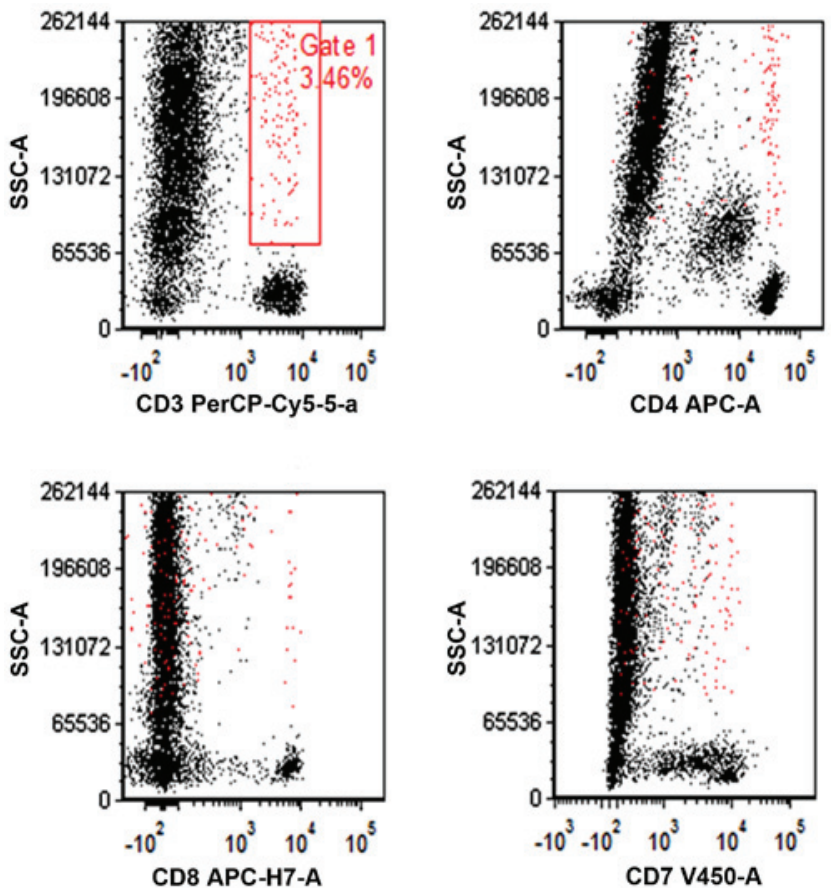

Figure 2. Artifacts on granulocyte and monocyte staining with lymphocyte markers. Representative dot plots showing the performances of CD3, CD4, CD8 and CD7 in a 8-color cocktail are shown. Gate 1 exhibits the artifacts on monocyte and granulocyte staining (red). The gate 1 population coexpressed multiple markers. Data representative of four samples is shown. CD, cluster of differentiation; APC, allophycocyanin; PerCP, peridinin chlorophyll protein complex; Cy-, cyanine; FITC, fluorescein isothiocyanate; SSC-A, side scatter area.
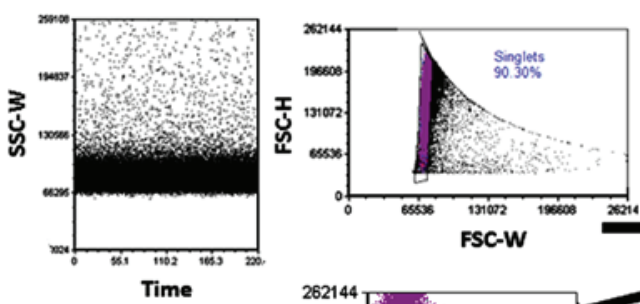
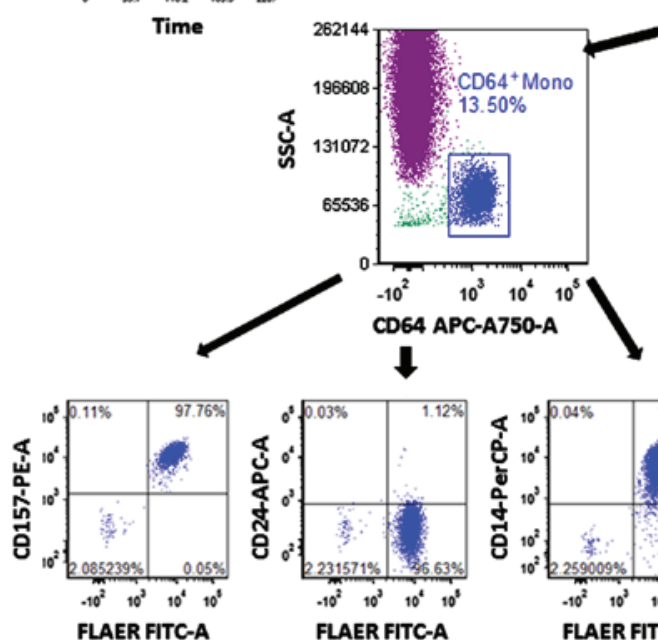

14
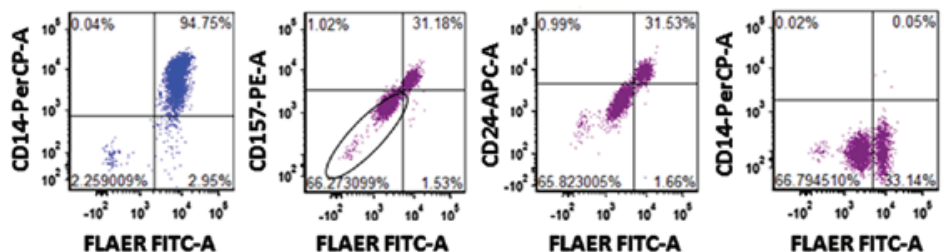

Figure 3. Gating strategy of 8-color paroxysmal nocturnal hemoglobinuria flow assay. A PNH ${ }^{+}$whole blood sample was stained with the 8-color PNH cocktail. Doublets were discriminated on a FSC-W/FSC-H plot. The monocytes and granulocytes were gated together on a CD45/SSC plot. The CD19-CD5- population was gated on a CD5CD19/SSC plot; non-specific binding on granulocytes and monocytes is indicated by the green arrow. The CD64 $4^{+}$Mono and CD15 $5^{+} \mathrm{Grans}$ were identified on CD64/SSC and CD15/SSC plots, respectively. The PNH clone was identified on CD157/FLAER, CD24/FLAER and CD14/FLAER plots as indicated in the black circle. Mono, monocyte; Grans, granulocyte; CD, cluster of differentiation; FLAER, fluorescein-labeled proaerolysin; PE, phycoerythrin; Cy-, cyanine; APC, allophycocyanin; PerCP, peridinin chlorophyll protein complex; FITC, fluorescein isothiocyanate; FSC, forward scatter; SSC, side scatter; -W, width; -H, height; -A, area.

cocktail containing FLAER-A488, PE-CD55, APC-CD14 and V500-C-CD45. From this, a minor CD55 granulocyte population was observed (Fig. 1). However, this population was FLAER ${ }^{+}$, suggesting that it was not representative of PNH 

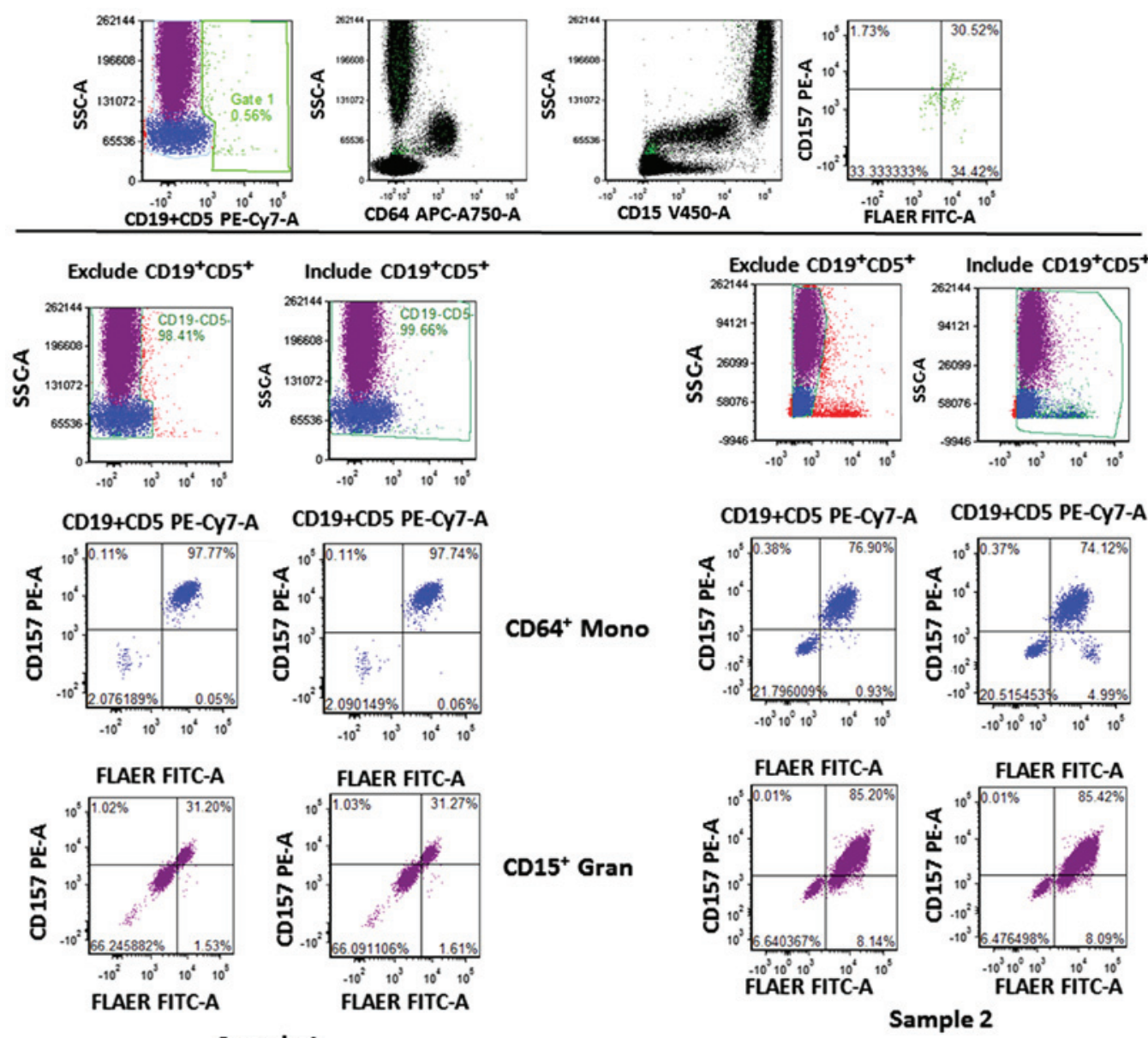

Sample 1

Figure 4. Gating strategy of 8-color paroxysmal nocturnal hemoglobinuria flow assay. The $\mathrm{CD}_{19} \mathrm{CD}^{+}$population was gated on gate 1 and its distribution was shown on the CD15/SSC, CD64/SSC and FLAER/CD157 dot plots, indicated in green (top panel). The percentages of FLAER/CD157 with exclusion (left) and inclusion (right) of $\mathrm{CD} 19^{+} \mathrm{CD}^{+}$cells in $\mathrm{CD} 64^{+} \mathrm{Mono}$ and $\mathrm{CD} 15^{+} \mathrm{Grans}$ from two samples (lower panel). Mono, monocyte; Grans, granulocyte; $\mathrm{CD}$, cluster of differentiation; FLAER, fluorescein-labeled proaerolysin; PE, phycoerythrin; Cy-, cyanine; APC, allophycocyanin; FITC, fluorescein isothiocyanate; FSC, forward scatter; SSC, side scatter; -A, area.

clones, which have been determined to be FLAER- (26). Three other normal samples exhibited the same FLAER-/CD55+ population of granulocytes (data not shown).

Artifacts on granulocyte and monocyte staining with fluorochrome-conjugated antibodies. To increase the sensitivity of multicolor flow cytometry, the present study aimed to remove non-specific binding and artifacts. A normal blood sample was stained with a multicolor cocktail targeting T cell markers (CD3, CD4, CD8 and CD7). A number of artifacts were identified in the granulocytes and monocytes regions on CD3/SSC, CD4/SSC, CD8/SSC and CD7/SSC plots (Fig. 2, indicated in red). Other lymphocyte markers including CD25 exhibited the same artifacts (data not shown). Some of those artifacts were doublets and could be eliminated by doublet discrimination (data not shown). However, some artifacts remained following gating out of all doublets as observed on the CD19+CD5/SSC plot in Fig. 3 (green arrow).

Granulocyte and monocyte PNH clones were identified with the 8-color flow assay. To determine whether the 8-color PNH panel was able to identify PNH clones, blood samples spiked with $\mathrm{PNH}$ clones were stained with an 8-color PNH cocktail (Table I). The forward scatter width and height (FSC-W and $-\mathrm{H}$ ) were used to gate the singlets population. Monocytes and granulocytes were gated together and non-specific binding events were removed by gating the negative population on the 'dump' channel (CD5 and CD19; Fig. 3). A number of artifacts were identified on the right side of the granulocyte and monocyte region on the CD5+CD19/side scatter (SSC) plot, and those events were eliminated by gating the negative populations of monocytes and granulocytes, resulting in reduced background (Fig. 3). The monocyte antigen CD64 and granulocyte antigen CD15 further separated monocytes and granulocytes, respectively. The PNH clones exhibited negativity for FLAER and GPI-linked antigens (CD157, CD24 and CD14; Fig. 3). The $\mathrm{CD}^{+} \mathrm{CD} 19^{+}$population was present together with $\mathrm{CD} 15^{+}$Grans, CD $64^{+}$Mono and affected FLAER ${ }^{-/ C D} 157^{-}$quadrant as indicated in Fig. 4 (top panel; green population). There were FLAER-/CD157- differences that ranged from 0.014 to $1.28 \%$ when excluding or including the $\mathrm{CD}^{+} \mathrm{CD} 19^{+}$populations (Fig. 4, lower panel). By contrast, normal whole blood stained with the same 8-color PNH cocktail did not exhibit any events on the lower left quadrant of FLAER/CD157, FLAER/CD24 and FLAER/CD14 plots (Fig. 5, top panel). The samples of 10 normal donors were 

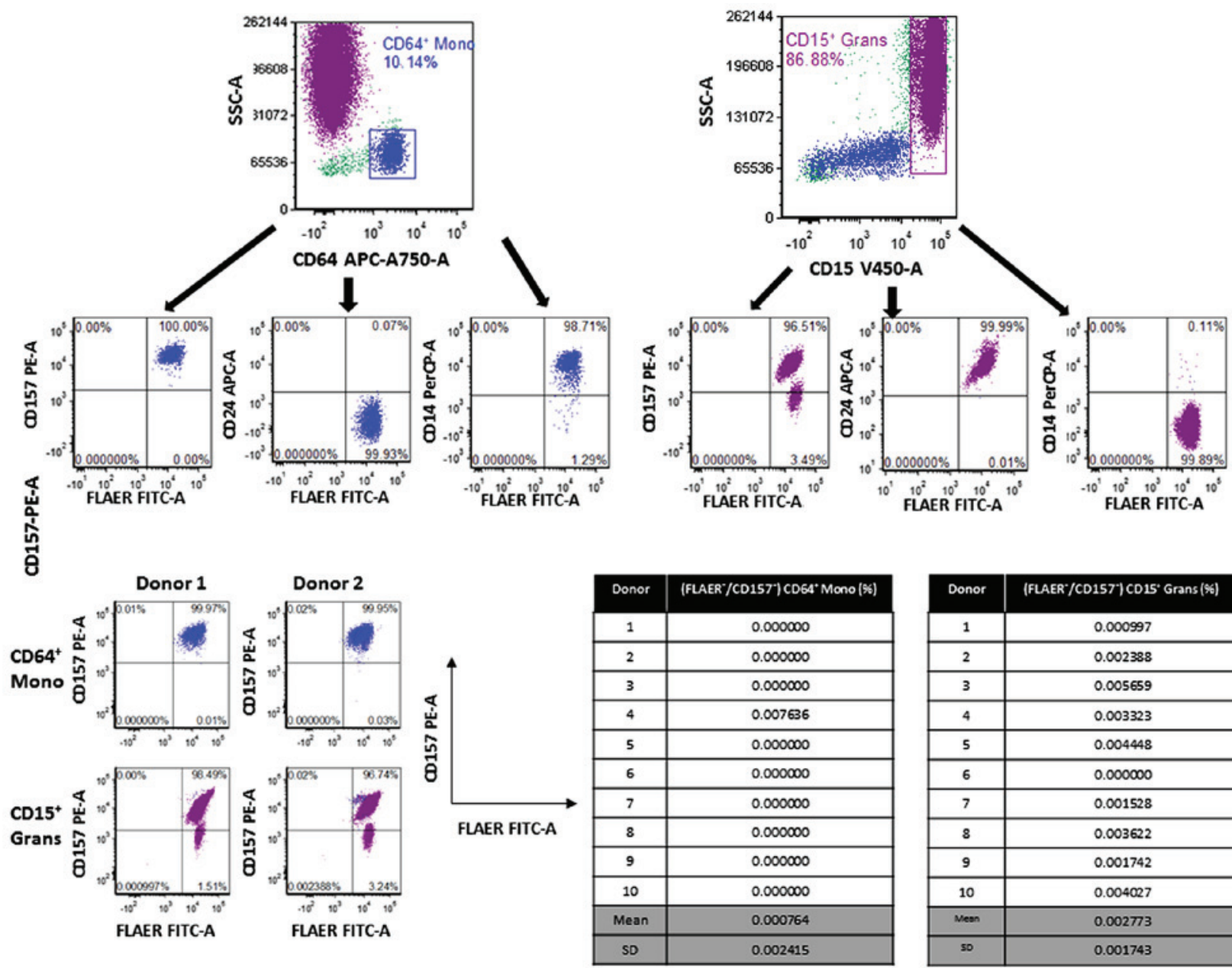

\begin{tabular}{|c|c|}
\hline Donor & (FLAER'/CD157) CD15' Grans (96) \\
\hline 1 & 0.000997 \\
\hline 2 & 0.002388 \\
\hline 3 & 0.005659 \\
\hline 4 & 0.003323 \\
\hline 5 & 0.004448 \\
\hline 6 & 0.000000 \\
\hline 7 & 0.001528 \\
\hline 8 & 0.003622 \\
\hline 9 & 0.001742 \\
\hline 10 & 0.004027 \\
\hline Meen & 0.002773 \\
\hline 50 & 0.001743 \\
\hline
\end{tabular}

Figure 5. Gating strategy of 8-color paroxysmal nocturnal hemoglobinuria flow assay. A normal whole blood sample was stained with the 8-color PNH cocktail as a control and PNH clones (FLAER $/$ CD157-) were not observed (upper panel). The representative dot plots from two normal donors (lower left) are shown $(\mathrm{n}=10)$. The table showing the percentage of PNH clones in CD15+ Grans and CD64+ Mono is shown (lower right). Mono, monocyte; Grans, granulocyte; $\mathrm{CD}$, cluster of differentiation; FLAER, fluorescein-labeled proaerolysin; PE, phycoerythrin; APC, allophycocyanin; PerCP, peridinin chlorophyll protein complex; FITC, fluorescein isothiocyanate; SSC, side scatter; -A, area.

stained with this 8-color PNH cocktail and exhibited an average of $0.002773 \pm 0.001743 \%$ PNH clones (FLAER $/$ CD157') in the $\mathrm{CD} 15^{+}$Gran population and $0.000764 \pm 0.002415 \%$ in the $\mathrm{CD} 4^{+}$monocyte population, respectively (Fig. 5, lower panel). These results suggest that the 8 -color assay has high specificity.

Sensitivity of the WBC 8-color PNH flow assay. The percentage of PNH clones in three PNH samples were determined using the 8-color panel. The PNH samples were then spiked into normal whole blood to attain a final PNH concentration of $0.5-2 \%$, and serial dilutions were performed. The first tube

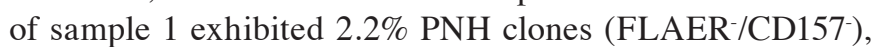
and was subsequently diluted in $50 \%$ increments until it reached $0.01 \%$ (Fig. 6). The normal whole blood control did not exhibit a PNH population on the left lower quadrant of the FLAER/CD157 plot (Fig. 6). The actual number of FLAER-/CD157- events in sample 1 was 10/69,216 $\mathrm{CD}_{15}{ }^{+}$Grans at the lower limit of detection (LOD) of $0.014 \%$ (Fig. 6 and Table III). The number of FLAER/CD157- events in samples 2 and 3 at the corresponding LODs were 10/73,469 CD $15^{+}$Grans and 8/77,229 $\mathrm{CD}^{+} 5^{+}$Grans, respectively
(Table III); samples 1-3 exhibited an LOD for FLAER $/$ $\mathrm{CD} 157^{-\mathrm{CD}} 15^{+}$Grans of $0.01 \%$ (Table III) and $0.05 \%$ for $\mathrm{CD}^{+}{ }^{+}$Mono (data not shown) on the 8-color flow assay. The expected PNH clone percentage was significantly correlated with the actual value, $\mathrm{R}^{2}$ value of 0.9839 and $\mathrm{p}$ value less than 0.0001 on a linear curve (Fig. 7), suggesting that the 8-color PNH flow assay had high sensitivity, detecting a PNH clone population as low as $0.01 \%$ in $\mathrm{CD} 15^{+}$Grans.

Accuracy of the WBC 8-color PNH flow assay. The percentages of PNH clones in the CAP and UK-NEQAS samples were determined using the 8-color panel. The percentage of FLAER-/CD157- Grans in the CAP sample was $8.79 \%$, which matched the CAP FLAER/CD157 granulocytes specification (4.70-10.00\%; Fig. 8). The percentage of

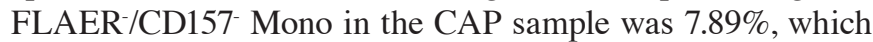
matched the CAP FLAER/CD157- monocytes specification (7.39-10.13\%; Fig. 8). Furthermore, the percentage of two UK-NEQAS samples identified by the 8-color assay matched the UK-NEQAS specifications (Fig. 8), suggesting that the 8-color flow assay had high accuracy for PNH clone identification. 


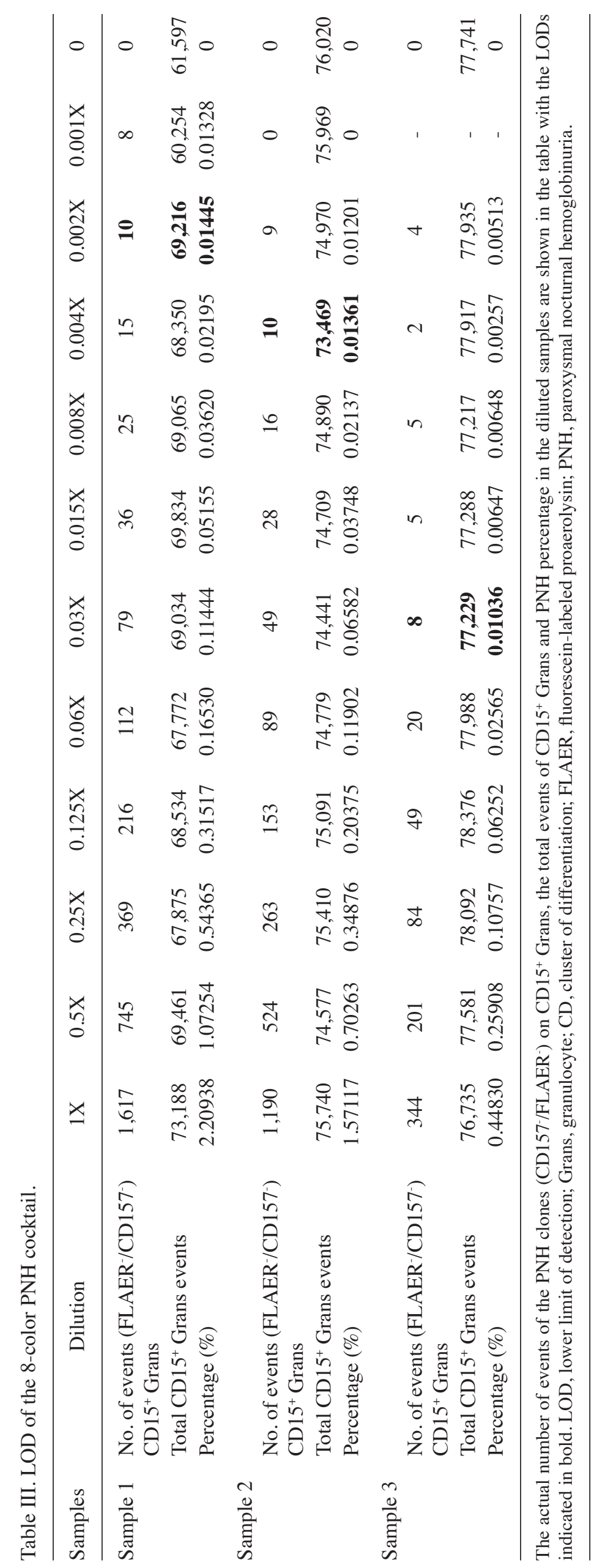



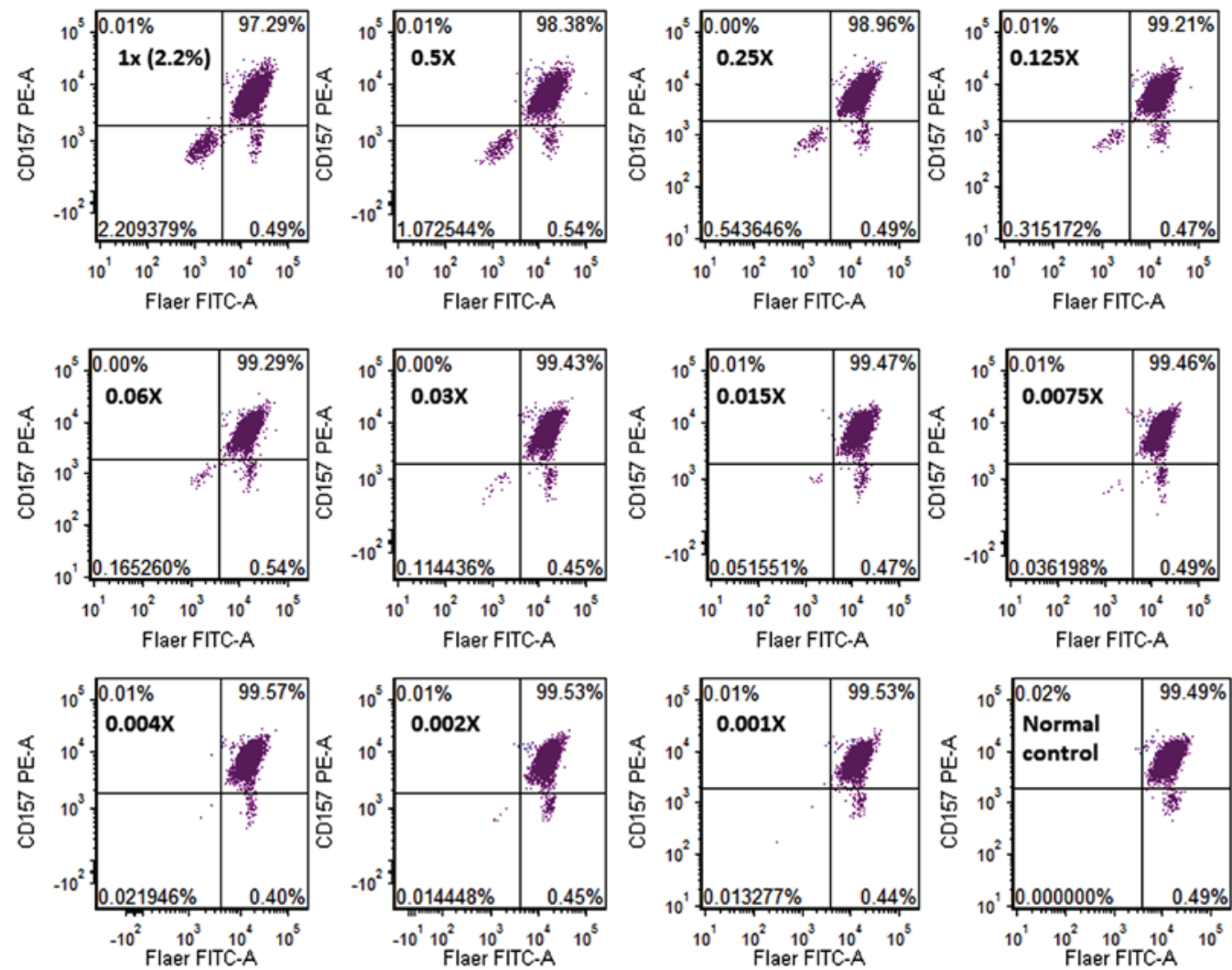

Figure 6. Sensitivity of the 8-color PNH flow assay. A PNH sample was spiked into a normal whole blood sample with a final PNH clone percentage of $0.5-2 \%$. The spiked sample was then serial diluted at (1:1) using the same normal whole blood as diluent up to 0.001X dilution. The percentage of PNH clones (CD157/FLAER gated on $\mathrm{CD}^{-} 5^{+} \mathrm{Grans}$ ) in each dilution is shown (dot plots of one representative sample are shown). A normal blood sample was used as a control. PNH, paroxysmal nocturnal hemoglobinuria; CD, cluster of differentiation; Grans, granulocyte; FLAER, fluorescein-labeled proaerolysin; PE, phycoerythrin; FITC, fluorescein isothiocyanate; -A, area.

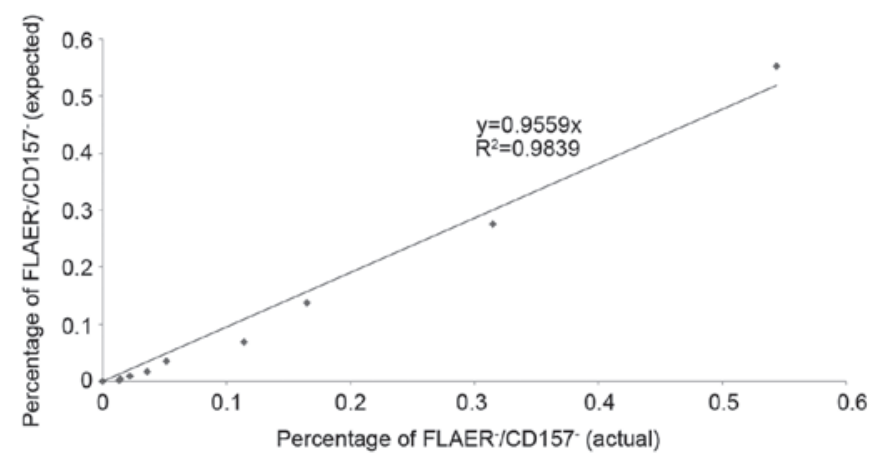

Figure 7. Sensitivity of the 8-color PNH flow assay. A linear correlation curve of expected PNH percentage compared with actual percentage was determined with $\mathrm{P}<0.0001$ (the results of one representative are shown). $\mathrm{PNH}$, paroxysmal nocturnal hemoglobinuria; $\mathrm{CD}$, cluster of differentiation; FLAER, fluorescein-labeled proaerolysin.

\section{Discussion}

Flow cytometry has become a standard tool for the analysis of cellular phenotype and function within the immune system, among other applications. As more fluorochromes have been developed with advances in cytometer design and lasers, the identification of more rare cell populations has become achievable, and the sensitivity of existing assays has increased $(1,2)$. For PNH, as a rare disease, a high sensitivity assay with short acquisition time is required.
In the present study, it was demonstrated that the 4-color combination (FLAER, CD55, CD14 and CD45) was not accurate in identifying $\mathrm{PNH}$ populations in whole blood samples. Earlier methods of PNH detection have relied on the loss of CD55 and CD59 on RBCs and granulocytes $(17,18,20)$. However, the present study identified that CD55 negative populations were not representative of $\mathrm{PNH}$ clones, suggesting that a novel assay is required to aid more accurate clinical diagnosis of PNH. Additionally, CD55 is not an adequate marker for PNH as it cannot distinguish PNH type II (partially deficient GPI-anchored proteins) from type III (lacking expression of GPI-anchored proteins) RBCs (20).

The improved methods in flow cytometry may enable the measurement of increased markers at a single cell level in less time $(2,29,30)$. In the present study, an 8-color configuration was designed for $\mathrm{PNH}$ identification. With increased photomultiplier tubes in the assay, it allowed the inclusion of other antigens that could increase sensitivity of the flow assay. However, artifacts were observed on monocyte and granulocyte surface staining even when using lymphocytes markers such as CD3 and CD7. These artifacts were composed of doublets and non-specific staining signals, which were present on the positive side of each channel. To generate a high sensitivity flow assay for PNH detection, the aim was to eliminate all potential non-specific binding events, as only 10 events in a 100,000 total population (granulocytes and monocytes) was required to achieve $0.01 \%$ sensitivity. A 

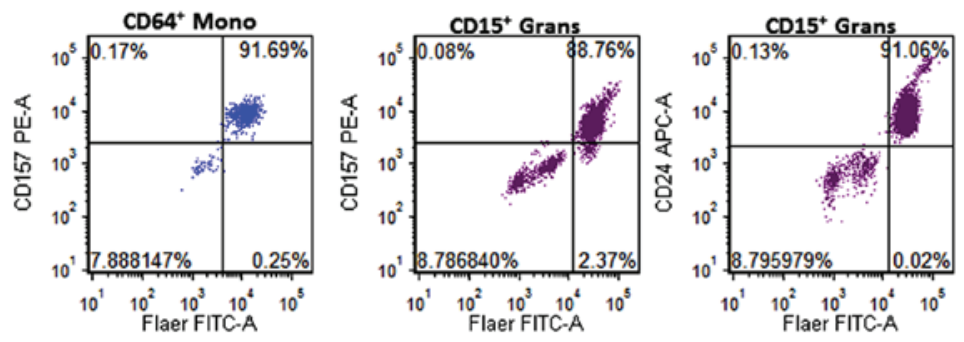

\begin{tabular}{|c|c|c|}
\hline \multirow{2}{*}{$\begin{array}{c}\text { CAP Sample } \\
1\end{array}$} & \multicolumn{2}{|c|}{ CAP Specifications } \\
\hline & Mean & $\begin{array}{c}\text { Reference } \\
\text { Range }\end{array}$ \\
\hline $\begin{array}{l}\text { FLAER } \\
\text { /CD157 } \\
\text { Mono }\end{array}$ & 7.59 & $4.7-10$ \\
\hline $\begin{array}{l}\text { FLAER } \\
\text { /CD157 } \\
\text { Grans }\end{array}$ & 9.05 & $\begin{array}{l}7.39- \\
10.13\end{array}$ \\
\hline \multirow{2}{*}{$\begin{array}{l}\text { UK NEQAS } \\
\text { Sample } 1\end{array}$} & \multicolumn{2}{|c|}{ UK NEQAS Specifications } \\
\hline & Mean & $\begin{array}{c}\text { Reference } \\
\text { Range } \\
\end{array}$ \\
\hline $\begin{array}{l}\text { FLAER } / \text { CD14 } \\
\text { Mono }\end{array}$ & 11.5 & $\begin{array}{c}10.43- \\
13.24\end{array}$ \\
\hline $\begin{array}{l}\text { FLAER /CD24- } \\
\text { Grans }\end{array}$ & 11.24 & $\begin{array}{c}10.56- \\
12.04\end{array}$ \\
\hline \multirow{2}{*}{$\begin{array}{l}\text { UK NEQAS } \\
\text { Sample } 2\end{array}$} & \multicolumn{2}{|c|}{ UK NEQAS Specifications } \\
\hline & Mean & $\begin{array}{l}\text { Reference } \\
\text { Range }\end{array}$ \\
\hline $\begin{array}{l}\text { FLAER /CD14- } \\
\text { Mono }\end{array}$ & 23.73 & $\begin{array}{c}18.34- \\
29.08\end{array}$ \\
\hline $\begin{array}{l}\text { FLAER /CD24- } \\
\text { Grans }\end{array}$ & 6.2 & $\begin{array}{c}5.45- \\
7.28\end{array}$ \\
\hline
\end{tabular}

Figure 8. Accuracy of 8-color paroxysmal nocturnal hemoglobinuria flow assay. The dot plots show the percentages of FLAER/CD157 and FLAER/CD24 clones gated on $\mathrm{CD} 15^{+}$Grans and FLAER/CD14 clones gated on $\mathrm{CD}^{+} 4^{+}$Mono from one CAP sample and two UK-NEQAS samples. The specifications of the reference samples are shown in the right tables. Mono, monocyte; Grans, granulocyte; $\mathrm{CD}$, cluster of differentiation; FLAER, fluorescein-labeled proaerolysin; PE, phycoerythrin; APC, allophycocyanin; PerCP, peridinin chlorophyll protein complex; FITC, fluorescein isothiocyanate; CAP, College of American Pathologists.

minority of false positive events would markedly decrease the sensitivity. The $\mathrm{CD}^{+} \mathrm{CD} 19^{+}$populations were combined with $\mathrm{CD} 15^{+}$Grans, $\mathrm{CD}^{+} 4^{+}$monocytes and were even identified on the lower left quadrant of the CD157/FLAER plot used for PNH clone identification. The percentage differences in FLAER $/$ CD157 clones by excluding and including $\mathrm{CD}^{+} \mathrm{CD} 19^{+}$populations were $0.014-1.28 \%$, which are significant numbers when considering a $0.01 \%$ PNH clone population was being targeted. However, it was demonstrated using doublet discrimination (FSC-W/FSC-H) and a 'dump' channel (CD5 and CD19) to gate the negative population that the background staining was lowered markedly to detect $0.002773 \%$ (CD15 ${ }^{+}$Grans) and $0.000764 \%$ (CD64 ${ }^{+}$Mono) $\mathrm{PNH}$ populations (FLAER-/CD157-) in whole blood from 10 normal donors. Sutherland et al (31) described a 4-color combination of FLAER, CD14, CD64 and CD45 for high-resolution detection of PNH monocytes with a background rate of $0.0033 \%$. Our current 8-color combination further improved the background rate for monocytes and granulocytes to $0.00076 \%$ and $0.00277 \%$, respectively, with only 100,000 events of monocyte and granulocyte acquisition. Furthermore, the gating strategy using granulocyte antigen (CD15) and monocyte antigen (CD64) served a key role in separating the monocyte and granulocyte populations prior to assessment of the GPI-linked antigens. As a result, the 8-color panel improved sensitivity and specificity with a shorter acquisition time in PNH identification.
FLAER-A488 is a fluorescent aerolysin that directly binds GPI-linked anchors and has been suggested as an optimum reagent for PNH assays (20). In the current study, three additional GPI-linked antigens were included to improve specificity. CD157 is a GPI-linked protein expressed on both granulocytes and monocytes (32); CD14 is a GPI-protein expressed on monocytes (27); and CD24 is a GPI-protein expressed on granulocytes (28). The combination of FLAER, CD157, CD24 and CD14 provided a comprehensive panel for targeting of all major GPI-linked antigens on PNH samples.

Furthermore, PNH samples provided by CAP and UK NEQAS were also tested by the 8-color PNH flow assay. The percentage of $\mathrm{PNH}$ clones identified by the 8-color assay matched the specifications provided by CAP and UK NEQAS, suggesting that the assay is able to identify $\mathrm{PNH}$ populations with sufficient accuracy.

In conclusion, flow cytometry is an important tool for leukemia/lymphoma diagnosis (33-36). Abnormal cells may exhibit aberrant expression of certain markers and downregulate other markers, in comparison to normal samples (37). It is important that a pre-mixed reagent generates an accurate expression profile of the sample using a combination of multicolor cocktails, to enable pathologists to correctly identify different types of leukemia/lymphoma and also PNH in patient samples (26,38-42). However, the current strategies employing CD55 and CD59-based assays in certain laboratories do not have adequate sensitivity to detect $\mathrm{PNH}$ population sizes of less than 
$1 \%$, and thus the $30-40 \%$ PNH samples that typically exhibit a lower than $1 \%$ PNH clone population go undetected $(21,43)$. A high sensitivity flow assay is required to identify those populations, to allow early and suitable treatments to be provided for patients. The present results demonstrate that the 8-color PNH antibody cocktail is sensitive in distinguishing small PNH populations from normal samples with low background and short acquisition time. It may provide an effective tool for pathologists in the diagnosis of $\mathrm{PNH}$, including in those cases with small percentages of PNH clones.

\section{Acknowledgements}

The authors are thankful to CAP and UK NEQAS for providing PNH samples for the study. All authors declare no conflicts of interest regarding the study described in this manuscript.

\section{References}

1. Chattopadhyay PK, Perfetto SP and Roederer M: The colorful future of cell analysis by flow cytometry. Discov Med 4: 255-262, 2004.

2. Chan RC, Kotner JS, Chuang CM and Gaur A: Stabilization of pre-optimized multicolor antibody cocktails for flow cytometry applications. Cytometry B Clin Cytom 92: 508-524, 2017.

3. Hill A, Platts PJ, Smith A, Richards SJ, Cullen MJ, Hill QA, Roman E and Hillmen P: The incidence and prevalence of paroxysmal nocturnal hemoglobinuria (PNH) and survival of patients in Yorkshire. Blood 108: 985, 2006.

4. Parker C, Omine M, Richards S, Nishimura J, Bessler M, Ware R, Hillmen P, Luzzatto L, Young N, Kinoshita T, et al; International PNH Interest Group: Diagnosis and management of paroxysmal nocturnal hemoglobinuria. Blood 106: 3699-3709, 2005.

5. Hosokawa K, Kajigaya S, Keyvanfar K, Qiao W, Xie Y, Townsley DM, Feng X and Young NS: T Cell transcriptomes from paroxysmal nocturnal hemoglobinuria patients reveal novel signaling pathways. J Immunol 199: 477-488, 2017.

6. Hill A, Ridley SH, Esser D, Oldroyd RG, Cullen MJ, Kareclas P, Gallagher S, Smith GP, Richards SJ, White J, et al: Protection of erythrocytes from human complement-mediated lysis by membrane-targeted recombinant soluble CD59: A new approach to PNH therapy. Blood 107: 2131-2137, 2006

7. Al-Ani F, Chin-Yee I and Lazo-Langner A: Eculizumab in the management of paroxysmal nocturnal hemoglobinuria: Patient selection and special considerations. Ther Clin Risk Manag 12: 1161-1170, 2016.

8. Fletcher M, Whitby L, Whitby A and Barnett D: Current international flow cytometric practices for the detection and monitoring of paroxysmal nocturnal haemoglobinuria clones: A UK NEQAS survey. Cytometry B Clin Cytom 92: 266-274, 2017.

9. Hill A, DeZern AE, Kinoshita T and Brodsky RA: Paroxysmal nocturnal haemoglobinuria. Nat Rev Dis Primers 3: 17028, 2017.

10. Brodsky RA: Paroxysmal nocturnal hemoglobinuria. Blood 124: 2804-2811, 2014

11. Nafa K, Bessler M, Castro-Malaspina H, Jhanwar S and Luzzatto L: The spectrum of somatic mutations in the PIG-A gene in paroxysmal nocturnal hemoglobinuria includes large deletions and small duplications. Blood Cells Mol Dis 24: 370-384, 1998.

12. Canalejo K, Riera Cervantes N, Felippo M, Sarandría C and Aixalá M: Paroxysmal nocturnal haemoglobinuria. Experience over a 10 years period. Int J Lab Hematol 36: 213-221, 2014.

13. Yamashina M, Ueda E, Kinoshita T, Takami T, Ojima A, Ono H, Tanaka H, Kondo N, Orii T, Okada N, et al: Inherited complete deficiency of 20-kilodalton homologous restriction factor (CD59) as a cause of paroxysmal nocturnal hemoglobinuria. N Engl J Med 323: 1184-1189, 1990.

14. Motoyama N, Okada N, Yamashina M and Okada H: Paroxysmal nocturnal hemoglobinuria due to hereditary nucleotide deletion in the HRF20 (CD59) gene. Eur J Immunol 22: 2669-2673, 1992.

15. Rother RP, Bell L, Hillmen P and Gladwin MT: The clinical sequelae of intravascular hemolysis and extracellular plasma hemoglobin: A novel mechanism of human disease. JAMA 293: 1653-1662, 2005.

16. Hill A, Richards SJ and Hillmen P: Recent developments in the understanding and management of paroxysmal nocturnal haemoglobinuria. Br J Haematol 137: 181-192, 2007.
17. van der Schoot CE, Huizinga TW, van 't Veer-Korthof ET, Wijmans R, Pinkster J and von dem Borne AE: Deficiency of glycosyl-phosphatidylinositol-linked membrane glycoproteins of leukocytes in paroxysmal nocturnal hemoglobinuria, description of a new diagnostic cytofluorometric assay. Blood 76: 1853-1859, 1990.

18. Hall SE and Rosse WF: The use of monoclonal antibodies and flow cytometry in the diagnosis of paroxysmal nocturnal hemoglobinuria. Blood 87: 5332-5340, 1996.

19. Brodsky RA, Mukhina GL, Li S, Nelson KL, Chiurazzi PL, Buckley JT and Borowitz MJ: Improved detection and characterization of paroxysmal nocturnal hemoglobinuria using fluorescent aerolysin. Am J Clin Pathol 114: 459-466, 2000.

20. Borowitz MJ, Craig FE, Digiuseppe JA, Illingworth AJ, Rosse W, Sutherland DR, Wittwer CT and Richards SJ; Clinical Cytometry Society: Guidelines for the diagnosis and monitoring of paroxysmal nocturnal hemoglobinuria and related disorders by flow cytometry. Cytometry B Clin Cytom 78: 211-230, 2010.

21. Movalia M and Illingworth A: Distribution of PNH clone sizes within high risk diagnostic categories among $481 \mathrm{PNH}$ positive patients identified by high sensitivity flow cytometry. Blood 120: $1271,2012$.

22. Hillmen P, Muus P, Röth A, Elebute MO, Risitano AM, Schrezenmeier H, Szer J, Browne P, Maciejewski JP, Schubert J, et al: Long-term safety and efficacy of sustained eculizumab treatment in patients with paroxysmal nocturnal haemoglobinuria. Br J Haematol 162: 62-73, 2013.

23. Sugimori C, Chuhjo T, Feng X, Yamazaki H, Takami A, Teramura M, Mizoguchi H, Omine $M$ and Nakao S: Minor population of $\mathrm{CD}^{-} 5^{-} \mathrm{CD} 59^{-}$blood cells predicts response to immunosuppressive therapy and prognosis in patients with aplastic anemia. Blood 107: 1308-1314, 2006.

24. Hillmen P, Lewis SM, Bessler M, Luzzatto L and Dacie JV: Natural history of paroxysmal nocturnal hemoglobinuria. N Engl J Med 333: 1253-1258, 1995.

25. Kelly RJ, Hill A, Arnold LM, Brooksbank GL, Richards SJ, Cullen M, Mitchell LD, Cohen DR, Gregory WM and Hillmen P: Long-term treatment with eculizumab in paroxysmal nocturnal hemoglobinuria: Sustained efficacy and improved survival. Blood 117: 6786-6792, 2011.

26. Sutherland DR, Acton E, Keeney M, Davis BH and Illingworth A: Use of CD157 in FLAER-based assays for high-sensitivity PNH granulocyte and PNH monocyte detection. Cytometry B Clin Cytom 86: 44-55, 2014.

27. Gatti A, Del Vecchio L, Geuna M, Della Porta MG and Brando B: Multicenter validation of a simplified method for paroxysmal nocturnal hemoglobinuria screening. Eur J Haematol 99: 27-35, 2017

28. Marinov I, Illingworth AJ, Benko $M$ and Sutherland DR: Performance characteristics of a non-fluorescent aerolysin-based paroxysmal nocturnal hemoglobinuria (PNH) assay for simultaneous evaluation of PNH neutrophils and PNH monocytes by flow cytometry, following published PNH guidelines. Cytometry B Clin Cytom: Jun 13, 2016 (Epub ahead of print).

29. Kusenda J, Fajtova M and Kovarikova A: Monitoring of minimal residual disease in acute leukemia by multiparametric flow cytometry. Neoplasma 61: 119-127, 2014.

30. Edwards BS, Oprea T, Prossnitz ER and Sklar LA: Flow cytometry for high-throughput, high-content screening. Curr Opin Chem Biol 8: 392-398, 2004.

31. Sutherland DR, Keeney M and Illingworth A: Practical guidelines for the high-sensitivity detection and monitoring of paroxysmal nocturnal hemoglobinuria clones by flow cytometry. Cytometry B Clin Cytom 82: 195-208, 2012.

32. Hernández-Campo PM, Almeida J, Sánchez ML, Malvezzi M and Orfao A: Normal patterns of expression of glycosylphosphatidylinositol-anchored proteins on different subsets of peripheral blood cells: A frame of reference for the diagnosis of paroxysmal nocturnal hemoglobinuria. Cytometry B Clin Cytom 70: 71-81, 2006.

33. Seifert RP, Bulkeley W III, Zhang L, Menes M and Bui MM: A practical approach to diagnose soft tissue myeloid sarcoma preceding or coinciding with acute myeloid leukemia. Ann Diagn Pathol 18: 253-260, 2014.

34. Mayson E, Saverimuttu J and Cartwright K: CD5-positive follicular lymphoma: Prognostic significance of this aberrant marker? Intern Med J 44: 417-422, 2014.

35. Pati HP and Jain S: Flow cytometry in hematological disorders. Indian J Pediatr 80: 772-778, 2013 
36. Krause DS, Delelys ME and Preffer FI: Flow cytometry for hematopoietic cells. Methods Mol Biol 1109: 23-46, 2014.

37. Huh YO and Ibrahim S: Immunophenotypes in adult acute lymphocytic leukemia. Role of flow cytometry in diagnosis and monitoring of disease. Hematol Oncol Clin North Am 14: 1251-1265, 2000.

38. Chung NG, Buxhofer-Ausch V and Radich JP: The detection and significance of minimal residual disease in acute and chronic leukemia. Tissue Antigens 68: 371-385, 2006.

39. Rawstron AC: Monoclonal B-cell lymphocytosis. Hematology Am Soc Hematol Educ Program: 430-439, 2009. doi: 10.1182/asheducation-2009.1.430

40. Del Principe MI, Maurillo L, Buccisano F, Sconocchia G, Cefalo M, De Santis G, Di Veroli A, Ditto C, Nasso D, Postorino M, et al: Central nervous system involvement in adult acute lymphoblastic leukemia: Diagnostic tools, prophylaxis, and therapy. Mediterr J Hematol Infect Dis 6: e2014075, 2014.

41. Hayashi D, Lee JC, Devenney-Cakir B, Zaim S, Ounadjela S, Solal-Céligny P, Juweid $M$ and Guermazi A: Follicular non-Hodgkin's lymphoma. Clin Radiol 65: 408-420, 2010.
42. Narita A, Muramatsu H, Okuno Y,Sekiya Y,Suzuki K, Hamada M, Kataoka S, Ichikawa D, Taniguchi R, Murakami N, et al: Development of clinical paroxysmal nocturnal haemoglobinuria in children with aplastic anaemia. Br J Haematol 178: 954-958, 2017.

43. Timeus F, Crescenzio N, Longoni D, Doria A, Foglia L, Pagliano S, Vallero S, Decimi V, Svahn J, Palumbo G, et al: Paroxysmal nocturnal hemoglobinuria clones in children with acquired aplastic anemia: A multicentre study. PLoS One 9: e101948, 2014.

(i) (9) This work is licensed under a Creative Commons Attribution-NonCommercial-NoDerivatives 4.0 International (CC BY-NC-ND 4.0) License. 